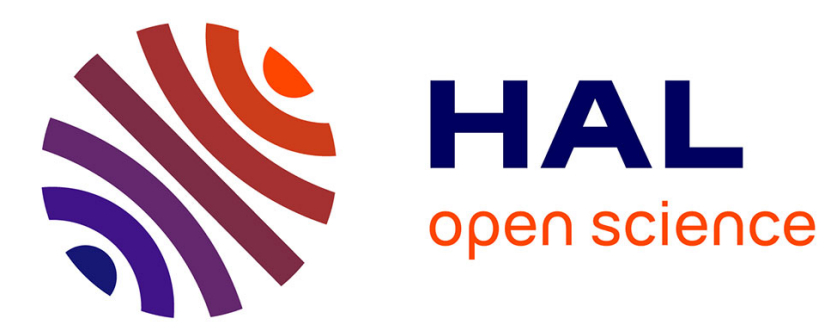

\title{
Le nain bossu au coq, de Strasbourg, et les Lagynophories alexandrines
}

\author{
Charles Picard
}

\section{To cite this version:}

Charles Picard. Le nain bossu au coq, de Strasbourg, et les Lagynophories alexandrines. Gallia - Fouilles et monuments archéologiques en France métropolitaine, 1958, 16 (1), pp.83-94. 10.3406/galia.1958.2226 . hal-01924535

\section{HAL Id: hal-01924535 \\ https://hal.science/hal-01924535}

Submitted on 3 Mar 2020

HAL is a multi-disciplinary open access archive for the deposit and dissemination of scientific research documents, whether they are published or not. The documents may come from teaching and research institutions in France or abroad, or from public or private research centers.
L'archive ouverte pluridisciplinaire HAL, est destinée au dépôt et à la diffusion de documents scientifiques de niveau recherche, publiés ou non, émanant des établissements d'enseignement et de recherche français ou étrangers, des laboratoires publics ou privés.

\section{(ㅇ)(1) $\$$}

Distributed under a Creative Commons Attribution - NonCommercial - NoDerivatives| 4.0 


\title{
LE NAIN BOSSU AU COQ, DE STRASBOURG, ET LES LAGYNOPHORIES ALEXANDRINES
}

\author{
par M. Charles PiCard
}

C'est une bien jolie découverte que M. J.-J. Hatt a faite à Strasbourg, et que Gallia (XII, 1954, p. 493, fig. 12) nous a donnéc à connaître.

Il s'agit d'une statuette en bronze, haute de $0 \mathrm{~m}$. 07 sculement, que des travaux de reconstruction ont permis de récupérer rue du Vieux-Marché-auxVins ${ }^{1}$. Elle représente (fig. 1) un nain bossu portant de la main gauche, non comme il a été dit - une "amphore", mais un lagynos alexandrin. Le petit personnage serre d'autre part contre lui, de son bras droit, un coq qu'il regarde, et qui a l'air d'essayer de le piquer avec son bec. - Lagynos et nanisme nous placent déjà dans un contexte assez alexandrin, notons-le d'emblée. M. J.-J. Hatt a vu justement que "les traits du personnage... et l'expression craintive sont rendus de façon caricaturale". Je serais moins d'accord avec lui pour ce qu'il a interprété sous le menton du nain comme une "double barbiche, assez longue, à l'instar des chèvres" dit-il' ; et des boucs, ajouterions-nous. Mais boucs et chèvres n'ont chacun qu'une barbiche. Par l'examen même de la figure 12 donnéc dans Gallia, on pouvait voir qu'il s'agit, au vrai, des deux exlrémités pendantes formant les brides d'attache d'un bonnet: bonnet assez collant, conique, dont la figurinc est coiffée. Ce que précise l'examen du moulage, et de l'original au Palais de Rohan.

Ce que peuvent confirmer aussi quelques autres exemplaires, plus ou moins analogues, dont nous disposons pour des comparaisons avec le document strasbourgeois. Ils étaient presque tous antérieurement connus; M. J.-J. Ilatt n'a pas manqué de rappeler, dans sa note de Gallia, l. l., leur existence ${ }^{3}$. Or le mieux conservé, provenant directement du Delta de Basse-Égypte, paruît pouvoir nous donner la localisation précise du thème traité, et aussi l'atelier de provenance : c'est assurément la région d'Alexandrie, où les compositions populaires - ne disons pas "grotesques" - avaient été mises à la mode dès

(1) M. J.-J. In et même un petit moulage en plătre patiné. Je lui exprime ici mon amicale gratitude.

(2) L. l., p. 493.

(3) P. 493, n. 5. 
le début de la période hellénistiquę. Le fait que le personnage est un nain, et difforme, et qu'il semble avoir un peu peur de son coq, presque aussi haut que son torse, fait penser à l'ambiance des petits villages du Nil, aux luttes des Pygmées contre les coqs el les grues du marécage, à l'extrémité des Bouches
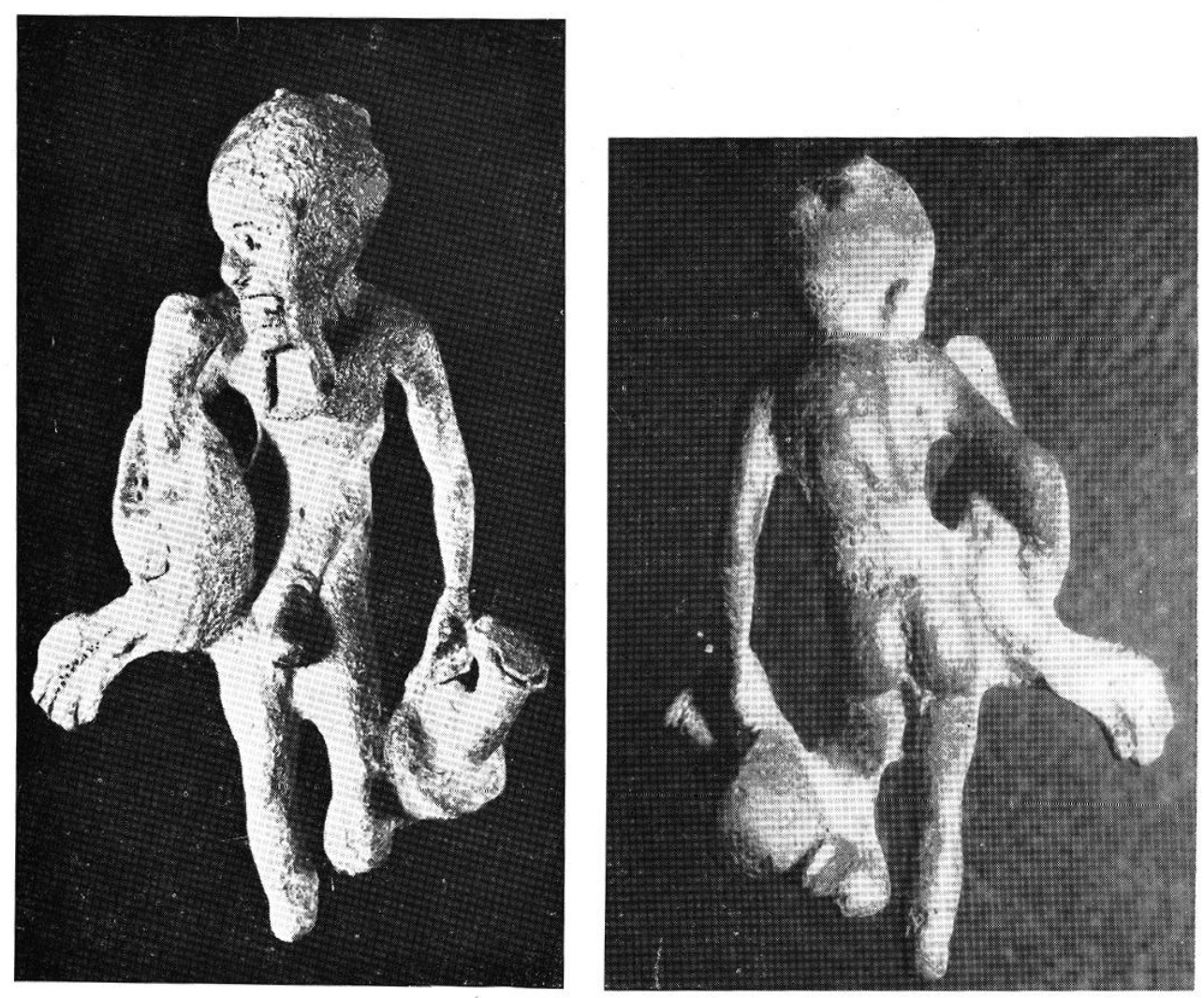

Fig. 1. - Le nain de Strasbourg (bronze). Haut. 0 m. 07.

du Nil'. Nous reproduisons (fig. 2, à g.) le document du cabinet du I) Fouquet, jadis commenté par P. Perdrizet, dans sa publication des Bronzes de la Collection

(4) Sur l'art populaire en Figypte alexandrine, sur les commencements d'une plastique compatissante aux humbles, aux disgrâciés de la nature, à l'humble classe des petits métiers des ports, de lá rue, ou des échoppes, cf. Ch. PrCard, Journ. psychol., 1951, p. 65-84: Sens et porlée des arts alexandrins. M. $\Lambda$. W. BYvanck qu'on n'accusera pas de faire à l'art alexandrin une trop belle part, reconnait au moins les sujets caricaturaux au compte de la cité des Lagides : Bullel. van de Vereeniging, 32, 1957, p. 13-33 ; cf. p. 13-14. Il regrette qu'il n'y ait pas de moyens (?), dit-il, pour mesurer l'expansion des bronzes du Delta. Cf. la statuette d'argent d'Alexandrie (British Mus.), qui représente un canard jouant avec un enfant assis, dont il cherche à mordiller l'oreille : p. ex., Max. Cor.lignon, Hisl. sculpt. grecque, II, p. 604, fig. 320 .

(5) Pour les Pygmées, nains du Sud de l'Égypte (ou de l'Inde ?), déjà mentionnés dans l'lliade (III, 3 sqq., : cf. Eustathe, ad. l.), cf. les dictionnaires de mythologie et d'histoire, de W. Roscher à 
Fouquel; car il s'agit là d'un petit bronze aussi', qui a à peu près les mêmes dimensions que celui de Strasbourg (ici, $0 \mathrm{~m}$. 075), le bas des jambes du nain étant aussi brisé et manquant. La patine vert foncé, luisante, correspond à celle de l'exemplaire de Strasbourg. La provenance indiquée est la Basse-Égypte. Voici la description de P. Perdrizet? : "Vieux pelit bossu tout nu, qui chemine d'un air inquiet et méfiant. C'est qu'il emporte de bonnes choses qu'on pourrait lui prendre : une amphore plcine de vin, et un coq, de quoi offrir un sacrifice à Asclépios, dicu de la santé, et après le sacrifice, de quoi bien boire el bien manger. Il tient l'amphore par l'une des anses (l'autre est cassée), et scrre son coq contre lui. De son menton pendent deux glandes, comme en ont les boucs... Noter le crâne rasé, sauf, en haut, une touffe de cheveux ".

De cette vivante présentation, on pense ici pouvoir corriger quelques détails. Le crâne n'est visible, ni rasé — "avec en haut une touffe de cheveux " car le personnage porte le même bonnet conique à brides et à protubérance en haut, sur le vertex, que le nain de Strasbourg, et que le joueur de castagnctles du groupe des petits danseurs de Canope, en bronze, repêchés à Mahdia; mais là, le bonnet est sans fanons. - D'autre part, ce que tient le nain n'est nullement - ici encore ! - une vraie " amphore ", mais un lagynos" ; celui-ci n'a jamais eu d'ailleurs qu'une anse, comme il fallait attendre.

P. Grimal, du P. W., RE., au Dict. Anl. .- Ph. E. I.rgrand, dans son commentaire d'Hérodote III 37 , néglige à tort l'existence d'une race naine antique au Sud de l'Égypte, et peut-être, d'autre part, en Phénicie (Pygmalion) : le nanisme n'est pas seulement une déformation tératologique, comme l'ont expliqué les médecins $\left(\mathrm{D}^{\mathrm{r}} \mathrm{P}\right.$. Richer, Nouvelle analomie arlistique: Le nu dans l'arl, L'art grec, 1926, p. 341-351, et déjà PARнor, Rec. de travaux de philol. et d'archéol. égyptienne et assyrienne, t. 2, 1880, p. 129 sqq.; Sur l'origine d'une des formes du dieu Plhah (sic!). On trouverait une bibliographie complétée dans l'étude de Gustaf Bergmark, Kungl. Velenskaps-Societelens Arsbok, 1951, Uppsala, 1952, p. 55 sqq. (Konsien som medicens förelöpare); cf. aussi Antonin Poncet et René Lericrie, Nains d'aujourd'hui el nains d'aulrefois, 1903. D'autre part, P. Montet, Rev. arch., 1952, II, p. 1 ssq. (Plah et les orfèvres) a montré qu'on choisissait, en Haute et Basse Égypte, les orfèvres des ateliers pharaoniques parmi les races naines. -... Le Sémite Pygmalion, qui passait parfois pour avoir fait lui-même la maquette, à Chypre, d'une statue de femme d'ivoire, dont il s'éprit avant d'obtenir d'Aphrodite de voir s'animer son "chef-d'ceuvre", n'aurait-il pas étè un Pygmée orfève et sculpteur? Cf. Marie Dricourt, Héphaistos ou la légende du magicien, 1957, p. 154, p. 162-163, p. 225.

(6) $\mathrm{N}^{\circ} 95$, p. 59 et pl. 28 en haut (deux aspects) ; Bronzes grecs d'Égupte de la Collection Fouquel, 1911. L'expression les "grotesques" encore si fréquemment employée vient de P. Perdizet, l. l., p. 59. Les références données par J.-J. Hatr auront à être révisées : 110, 495 ne correspondent à rien, les Bronzes Fouquet n'ayant ni 110 p., ni surtout 495.

(7) Cf. Gabriel Ifroux, Lagynos, 1913 (thèse complémentaire de Sorbonne) : en frontispice, copie de la statue de la "vieille femme ivre assise ", en marbre, de Munich, qui presse sur son cour un lag:ynos (?); l'original est attribué traditionnellement à Myron de Thèbes (niI ${ }^{\mathrm{c}}$ s. av. J.-C. (?); mais il n'était peut-être pas l'auteur de la "Vieille femme ivre" de Smyrne, dont a parlé Pline, Nat. hist., 36, 33 : contre-sens possible sur le nom de Maronis. On a sans doute une épigramme concernant l'une des ceuvres de Myron à Olympie; des signatures (discutées à tort) sont conservées à Pergame et à Rome; cf. Weissin̈upl, Arch. Ephem. 1891, p. 151. - Sur Myron de Thèbes, cf. principalement G. Liproln, Die griech. Plastik, 1950, p. 322 et pl. 112, $2:$ date incertaine. 
Ainsi en décide - plus nettement encore que sur le petit bronze trouvé par M. J.-J. Hatt - la "cassure " prononcéc, à l'épaule du vase, pour laquelle les exemples comparables ne manquent guère dans la séric abondante des lagynoi. C'est même là l'élément caractéristique de la morphologie du récipient alexandrin, pour la plupart des pièces encorc conservées, dont Délos a fourni un bon lot; Priène, Théra et Pergame, aussi. Ces produits de la céramique hellénistique forment, de l'un à l'autre centre, une séric homogène, bien connue, qui commence à l'époque des premiers Lagides; elle a été imitée jusqu'à Carthage ${ }^{8}$, peut-être jusqu'cn Gaule ${ }^{9}$. La fabrication a duré assez longtemps, tant en Égypte qu'ailleurs ${ }^{10}$. Les formes ont naturellement varié çà et là, mais on reconnaît généralement, d'emblée, ces vases -bouteilles, même brisés, à la largeur de leur panse, à la hauteur de lcur col, d'où se détache généralement une scule anse coudée $e^{11}$. Certains ont été décorés de relicfs appliqués à la barbotine ${ }^{12}$; la plupart, de peintures sur fond chamois.

Je ne pense pas que P. Perdrizet ait été bien inspiré en songeant aux apprêts d'un sacrifice à Asclépios. Il eût été étrange que l'offrande du coq fût remportée sitôt après le sacrifice, par le donateur, ce qui n'eût convenu ni au dieu de la santé, ni à son clergé, qui lui aussi aimait le poulet. D'autre part, le IVe mime d'Hérondas suffirait à faire connaître qu'on n'offrait pas à Asclépios du vin, avec le coq : oiseau dont Fr. Cumont, notamment, a montré la valeur religieuse

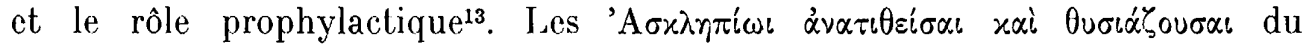
mime savamment localisé à Cos par le poète Ilérondas aux premières années du III $^{\mathrm{e}}$ s. av. J.-C., reçoivent bien du sacristain de l'Asclépieion - remises ensuite à Coccalé - certaines pièces de la victime dépecée, dont le sacrificateur avait dû couper le col, selon la mode ancienne et encore actuelle; mais la cuisse de la volaille revient — on nous le dit - de droit, au petit personnel sacerdotal, et elle lui est remise, en fait, après amputation ${ }^{14}$.

(8) Askos alexandrin du Musée Alaoui à Tunis, trouvé au Cap Bon : Ch. Picarn, Rev. arch., 1951, I, p. 215-217 (Nécropole de Sidi Djemaïl ed Dine); cf. Gilbert PICARD, Le monde de Carlhage, 1956, pl. 45 (en bas) : technique des lagynoi.

(9) Fragments du Musće de Pithiviers : fouilles de Sceaux; cf. ci-après.

(10) Une imitation tardive de lagynos, inédite, au Muscée du Caire, sera prochainement publiée à ma demande, dans les Mon. Piot, par M ${ }^{\mathrm{mc}}$ L. Ghali.

(11) G. Leroux, Lagynos, fig. des p. 15 à 68 ; cf. aussi P. Grarndor, Terres-cuites de l'Égypte gréco-romaine, 1939, p. 175-177. la p. 137).

12) P. ex. celui d'Athènes (Mus. nat. $2170: n^{\circ} 137$ du catalogue de G. Leroux, à la p. 68, fig. à

(13) CRAI., 1942, p. 284 sqq. et Le symbolisme funéraire, p. 398.

(14) V. 79-85. De même, les gâteaux préparés par Coccalé pour être offerts au dieu sont bien restitués à la jeune femme après exposition temporaire sur la trapeza des offrandes; mais c'est pourqu'ils soient distribués à l'assistance; ainsi, l'on offrait autrefois dans nos églises le pain bénit; les gâteaux consacrés préservaient de la malchance. 
Le nain bossu, représenté avec son coq et sa bouteille, aurait risqué de faire trop maigre chère, s'il avait eu l'idée de passer d'abord pour une offrande au sanctuaire de quelque génie guérisseur.

Achevons enfin la reprise critique de l'exégèse présentée par P. Perdrizet, et notons qu'il s'est trompé aussi, en pensant que "du menton " du personnage de la Coll. Fouquet "pendent deux glandes, comme en ont les boucs ». I.es boucs n'ont jamais eu de glandes caprines si longues que ... les brides, ici reconnaissables, du bonnet, lui-même visible.

Un autre fait doit être noté encore. Si nous avons trois petits bronzes de Lagynophores déjà - car il en a été signalé aussi un à Florence, que je n'ai pu étudier -, il se trouve que personne n'a reparlé jusqu'ici d'un vase de terrecuite entrant dans la série; il est pourtant connu, grâce à P. Graindor, qui l'avait fait entrer dans sa collection ${ }^{15}$. Il l'a décrit, malheureusement sans le reproduire, dans son dernier travail, publication posthume, imprimée sans ses vérifications, en 1939 : sous no 84 , p. 175-177. Il s'agit d'un esclave, probablement sémitique ; agenouillé sur le sol, il portait un lagynos, dont sa main gauche tenait l'anse. La tête est barbue, et elle est coiffée de longs cheveux, retombant à l'arrière jusqu'à la naissance du dos. L'œil est incisé d'un point, marquant l'iris. On se reportera à la description de P. Graindor, faute d'image. Relevons ici un délail que le savant archéologue belge avait noté : après le temps des lagynoi hellénistiques, on employa en Égypte des gourdes à reliefs d'appliques,

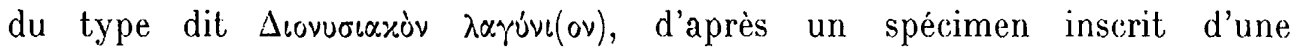
ancienne collection privée du Caire (Arch. Jahrb., Anz., 44, 1929, p. 204 sqq.). P. Graindor avait signalé aussi que les vases de ce type comportaient fréquemment la représentation de couples de nègres. C'est dire qu'ils étaient destinés par le commerce, en Égypte, au pctit pcuple indigène de couleur. Il n'est pas douteux, bien entendu, qu'on ajoutera peu à peu à la documentation utiliséc ici ; le thème du nain ou du nègre lagynophore a du être assez en faveur.

Si l'humble Pygmée d'Alexandric ne se dirige certainement pas vers un temple d'Asclépios, où donc pensait-il aller "bien boire et bien manger"? Nous le pouvons savoir, semble-t-il. Le culte dionysiaque, qui, à partir de l'époquc hellénistique, bénéficia d'une voguc exceptionnclle, au Delta d'Égypte, comportait des fêtes populaires ; elles ont, d'ailleurs, contribué à son expansion ${ }^{16}$. Ptolémée IV Philopator (221-203) avait institué dans sa capitale, une frairie plus ou moins copiée sur celle des Choes qu'on célébrait à Athènes ; on l'appelait

(15) Ci-dessus, n. 11

(16) G. Leroux, Lagynos, p. 79. 
à Alexandrie Lagynophorie ${ }^{17}$. L'usage était, lors de cette cérémonie, de célébrer un banquet populaire, où chacun apportait, son lagynos. Il est probable que dans plus d'une ville et à la campagne, on imitait cette liesse particulièrement, agréable aux humbles. Avec leur anse robuste, leur large panse bien fermée,

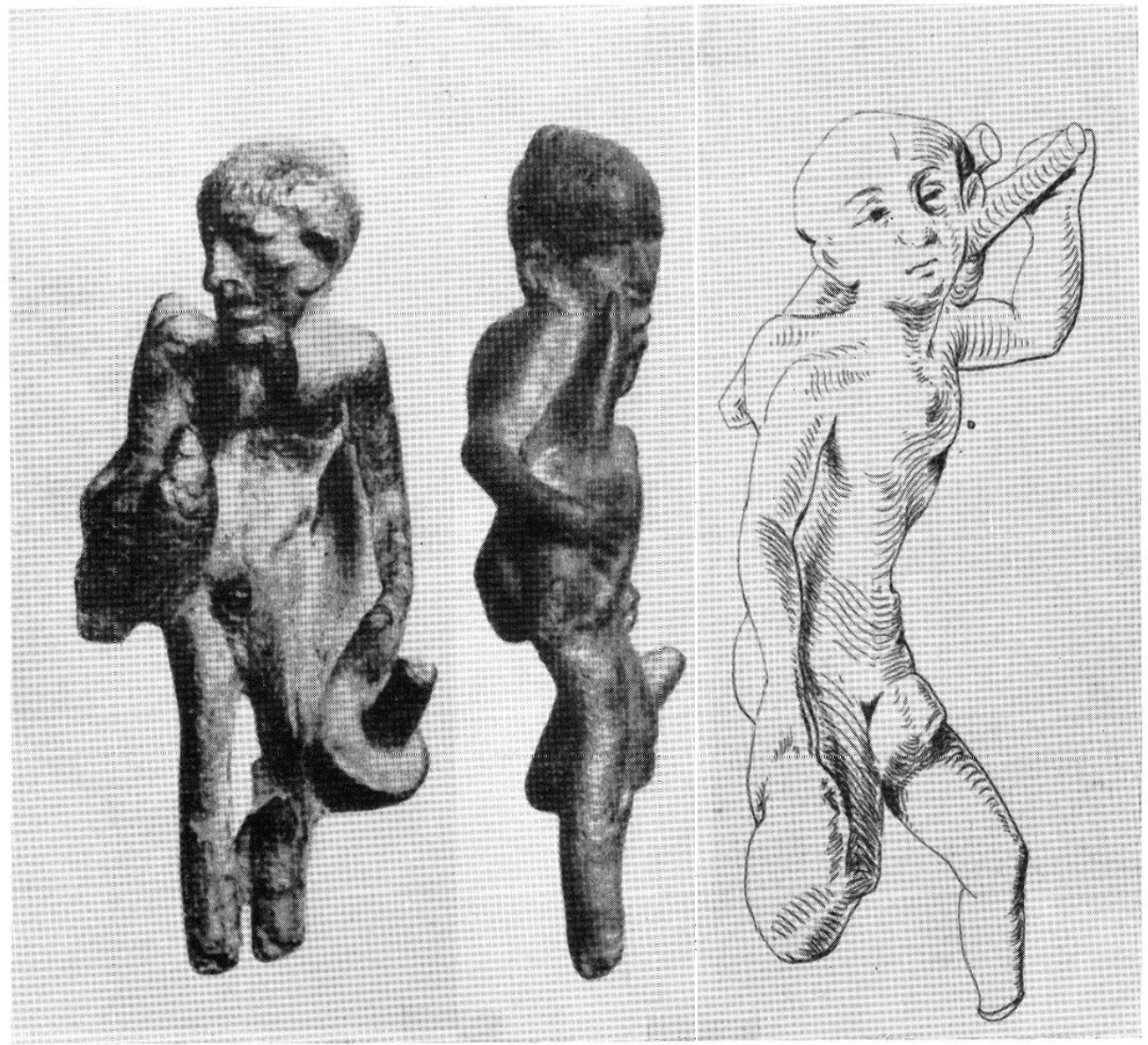

Fig. 2. - Bronze de la Collection Fouquet, Le Caire.

Fig. 3. - Nain porteur d'amphore, de Vienne (Autriche).

leur ornementation volontiers bachique ${ }^{18}$, les lagynoi étaient faits pour se substitucr, en ces occasions, au $\chi 0 u \tilde{s}$ des Attiques, l'œnochoé rituelle lors du

(17) A ArIÉNÉE, Banq. Soph., VII, 276, 6 (d'après Eratosthénès de Cyrène). En général, Von Gersau, Lagynophorie, col. 465 ; cf. M. P. Nilsson, Griech. Feste, p. 468 ; P. PERdrizet, Bull. Soc. archéol.

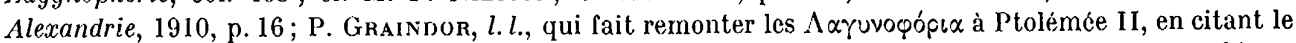

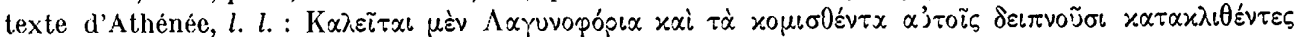

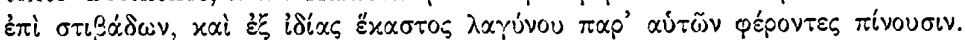

(18) C'est le cas sur le beau lagynos d'Athènes (ci-dessus, p. 86, n. 12) no 137 du Catalogue de G. Leroux, qui portait sur son épaule cinq groupes de figures en relief, montées à part et collées à la 
banquet sacré des Anthestéries, et sur laquelle, au retour, on posait une couronne de feuillage, symbole-souvenir de la réunion ${ }^{19}$. On pourrait reconstituer, le cortège des comastes, avec leurs lagynoi aux vives couleurs, parés de guirlandes de lierre au naturel : ornement qu'on voit, en fait, surtout imité au méplat de l'épaule ${ }^{20}$. Le lagynos, nous a dit Athénée $e^{21}$, valait douze cotyles attiques, c'est-à-dire près de deux litres et demi; mais on connaît un lagynos rpíxo'ss, dont le contenu était d'environ huit litres, sans doute pour des piques-niques collectifs (?). Les vases retirés du vaisseau qui a sombré vers Anticythère, et celui que tient en son giron la "Vicille femme ivre", altribuéc à Myron (de Thèbes), avaient une capacité plus grande ${ }^{22}$. Pour les préserver des chocs pendant le lransport et l'orgie, on les entourail parfois de paille tressée, comme on fail encore pour le fiasco de Chianti. Une mosaïque du musée Alaoui représente un excmplaire de cette sorte, avec sa garniture tresséc ${ }^{23}$. Ces flacons ont été en vogue, et répandus au loin, en raison de leur commodité, mais surlout des bons crûs qu'ils permettaient d'exporter ${ }^{24}$.

A la fête des lagynoi d'Alexandrie, on ne se contentait pas loujours d'ailleurs, scmble-t-il, de récipients restés de capacité modeste. Un petit bronze du Musée de Vienne, qui ne m'est connu que par un dessinin, se classe à part, car il reproduit cette fois - seul jusqu'ici à ma connaissancc - une grande amphore, du type de celles qu'on retrouve à Châteaumeillant (Cher) par excmple ${ }^{26}$. C'est toujours un petit nain bossu qui la transporte, et bien pleine, car celte fois, en raison de la lourdeur, il la tient à l'épaule (fig. 3). Malheureusement, nous ne pouvons pas décider de ce qui est figuré à la main droite: on croit reconnaître une sorte de sac (?) à la place du coq ou poulet. En marche, la tête détournée, le joyeux nain n'a pas l'air accablé par le fardeau temporaire de son amphore pointue. Maigre, il est affligé de deux bosses, cette fois, une par devant, une

barbotine : des Silènes y paraissaient, dont l'un titubant; on voit là aussi une Ménade qui danse en agitant ses crotales.

(19) P. Foucart, I.e culle de Dionysos en Allique, p. 116 sqq. ; E. Pruht, De Alheniensium pompis, p. $62-72$; I. DEUBNER, Allische Feste, 1932, p. $93-151$, pl. 8 sqq.

(20) Sur ces transpositions hellénistiques, sur leur rôle dans le développement de l'illusionisme alexandrin, cf. G. Leroux, l. l., p. 119 sqq., et. dẹjà p. 78-80 ; cf. aussi l'intaille en cornalinc reproduite par A. Furtwängler, Antike Gemmen, pl. 46, 24 ; ibid., t. II, p. 222.

(21) Cf. G. Leroux, l. l., pour ces mesures.

(22) Je n'en connais pas, sauf erreur, qui proviennent des cargaisons naufragées près de la côte de Provence.

(23) Catal. du Musée Alaoui, X, Suppl. 1907, p. 23, n० 279, pl. 15, nº6.

(24) G. Leroux, l. l., p. 82 sqq.

(25) Ed. von Sacken, Die antiken Bronzen des K. u. K. Münz-u. Anliken Cabinettes in Wien, 1871 , p. 118 , pl. 46-6 ; il n'est pas exact, comme l'avait dit P. Perdrizet, que le document ait été mal reproduit dans S. Reinach, Rép. stal., II, 566, n. 2 : le dessin publié là est correct (ici, fig. 3).

(26) Cf. E. Curénon, Châleaumeillant; les fouilles actuelles multiplient leur nombre. 
autre saillante à l'arric̀re. Sur la tête, E. von Sacken avait bien reconnu la "Zipfelmützc », mais sans fanons, au-dessus du petit visage pointu ("spilzig »), aux grandes oreilles décollées. Le bronze de Vienne, aussi, est brisé aux jambes.

La démarche vive du petit personnage est significative ici, non moins que son visage de gavroche urbain. Il va à la fête. Nous avons, grâce à Athénée, les moyens de le suivre, par la pensée au moins. Les Lagynophories, à Alexandrie comme ailleurs, n'étaient pas des plaisirs auliques; rien de commun avec les

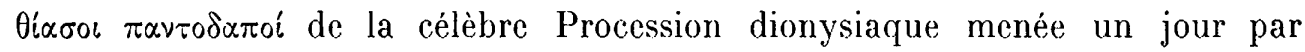
Ptolémée II, à travers Alexandric ; il s'agissail, le plus souvent, de bombances modestes de pauvres gens, méprisées plus ou moins tacitement à la cour, la Regia bénéficiant de cérémonies plus aristocratiques. (Chacun apportait son repas : il n'y a pas lieu de croire, avcc P. Graindor, que tous les nains bossus de la collection Fouquet, ou de Strasbourg, ou d'ailleurs, étaient sculement des esclaves d'accompagnement ${ }^{27}$. Pour des réunions de riches, ils auraient dû fournir bien autre chose qu'un coq, non encore plumé et vidé, et qu'une modeste bouteille. - Lors du repas, on s'étendait à la bonne franquette sur des jonchées de feuillage - failes par préférence et déférence de lierre bachique, si possible ; tels ont été les slibadeia primitifs ${ }^{2 \star}$. Il ne devait pas manquer par terre, après la partie, de détritus gênants. A ce sujet, un texte d'Érathosthène de Cyrène a laissé entendre quelque chose, dans un ouvrage intitulé Arsinoé (il semble qu'il s'agisse d'Arsinoé III, et non de l'Arsinoé Philadelphe ${ }^{29}$. Cette $\Lambda$ rsinoé, princesse de haut rang, aurait interrogé un jour - d'après F́rathosthène, qui vécut à la cour d'Alexandrie de Ptolémée III à Ptolémée $\mathrm{V}$, - un homme

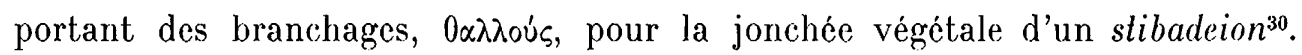
La rcine voulait savoir de quelle fête il s'agissait au juste. On lui répondit que c'était la Lagynophorie ${ }^{31}$. Quand l'homme cût parlé et qu'il se fût écarté, Arsinoé aurait tourné les yeux vers sa suite, disant avec quelque dégoût : "Voilà des lieux qui vont être sans doute bien malpropres, car il ne viendra apparemment

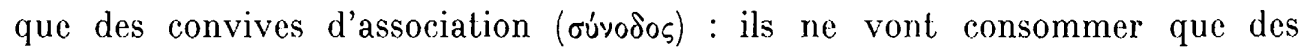

(27) I. l., p. 176. Cela pourrait être, tontefois, le cas pour le porteur du musce de Vienne.

(28) Ch. PICARD, Comptes rendus Acad. Insc., 1944, p. 127-157.

(29) Cf. E. Brvan, Hisl. des Lagides, p. 268-269, et J АCовY, Fragm. hist. graecorum, p. 713, n० 241, ont, semble-t-il, fait erreur sur ce point; cf. Müllek, Bühnen, p. 382 ; M. P. Nılssox, Griech. Feste, p. 486 ; MAн AFFY, Plolem. Dynasly, p. 161.

(30) Ce serait une erreur absurde de vouloir corriger, comme a fait Stichle, en $\varphi \alpha \lambda \lambda$ oús. Pourquoi, en ce cas, la question d'Arsinoé, et ce pluriel d'encombrement ? $\mathrm{A}$ Cos, au $\mathrm{I}^{\mathrm{er}} \mathrm{s}$. av. J.-C., Dionysos est

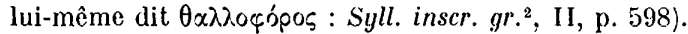

(31) Sur le personnage d'Ératosthénès, cf. Kisack, Eralosthenes, col. 358-387 (principalement, p. 360). Il avait composé, peut-on croirc, son Arsinoé après la mort de Philopator : Susemili, L.ill. alex, I, p. 409-428; J. Beloch, Griech. Gesch., IV, 2, p. 596-598. 
viandes rances et dégoûtantes $)^{32}$. C'était mépriser de haul, et les participants, et leur repas. - Plaisirs populaires, dirons-nous, cn tout cas, donl l'évocation, à côté des grandes pompes urbaines et des navigations fastueuses des thalamèges officielles sur le Nil, n'est que plus sympathique ${ }^{33}$.

Pcut-on croire que nous en ayons fini, ainsi, avec le Iagynophore de Strasbourg? Je ne le pense pas, car le plus intéressanl est sans doute de tenter d'expliquer ce que l'image alexandrine d'un nain d'Egyple allant faire bombance pouvail avoir comme attrait - exotique ? -... sur les bords du Rhin. Je crois que l'objet appartenait à un Alexandrin immigré. Car ne serait-il pas trop simple d'invoquer seulement le goût personnel d'un quelconque amatcur local de bibelots d'art? Il y a eu jadis, ici et là, d'autres collectionneurs antiques de curiosités alexandrines; probablement beaucoup dans les Balkans et dans l'Italic du Nord : les trouvailles de Florence, de Vienne (Nutriche), nous l'indiquent déjà et leur nombre a chance de s'augmenter. Je pense toulefois que la découverte de M. J.-J. Hatt, à Strasbourg, présente un intérêt šécial touchant l'histoire du commerce antique de Rhénanie, cl que pour l'ethnographie, d'autre part, nous avons là l'avantage d'une provenance assez inslructive. M. J.-J. Hall a déjà prouvé heureusement que la ville du Bas-Rhin devait contenir au moins un embryon de colonic alexandrine, sous l'Empire. Dans lc fascicule même de Gallia où il a fait connaitre le nain au coq, il a montré aussi forl à point une lampe égyptisante, trouvéc en ville, à $\Lambda$ rgentorate, dans un riche dépotoir de lit de rivière ${ }^{34}$. Il s'agit de la belle lampe à deux becs "décorée de masques tragiques", qui avail été signée par le potier romain Catullus. Or, Calullus travaillait là d'après des modèles alexandrins; car les pseudo-«masques tragiques» - qui n'ont pas eu la bouche grande ouverte, tant s'en faut! - ne sont rien d'autre que des visages égyptisants; ils sont caractéristiques sur les lampes d'Alexandrie que l'on connait : jusqu'au Maroc (Banasa), jusqu'à $\Lambda$ quileja (Italie du Nord), jusqu'à Byblos, el un peu partout ailleurs. Je crois avoir rétabli la provenance originelle, indûment cherchée parfois du côté de l'lbérie, de l'Étrurie ${ }^{35}$.

(32) Ici se place le passage transcrit plus haut, p. 88, n. 17.

(33) Sur l'Arsinoé, cf. K×AAGK, l. l., col. 386 ; Susemin, l. l., p. 422; E. BEvaN, Hisl. des Lagides,

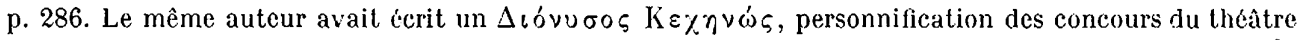
et des acleurs (masque à bouche larement béante); cf. Powlict, coll. Alexandrina, p. 65, fragm. $28^{\mathrm{b}}$. Pour plaire a la cour, Fratosthènis, on le voit, traitait abondamment des sujets dionysiaques ; ajoutons la mention de son Erigoné (fille d'Icarios) : KNAACK, $l$. $l$., col. 384-388.

(34) Gallia, XII, 1954, p. 329-330, fig. 11 à la p. 334.

(35) Rev. archéol., 1955, I, p. 63 sqq. (à propos des lampes égyplisantes de Volubilis et Banasa). 
Nul doute d'ailleurs qu'Argentorate puisse livrer encore d'autres vestiges de l'industric du Delta : déjà connus, ou qui sortiront peu à peu de terre. On a signalé p. ex. un balsamaire, à étudier, de provenance égyptienne. Je pense pour ma part que la petite colonie alexandrine qui vivait aux bords du Rhin devait être active et prospère. On connait une des raisons qui avaient attiré de ce côté les vaúxinpor et pilotes du pays du Nil : on avait eu besoin d'eux

Fig. 4. - Nègre du musée de Pithiviers, Loiret ( ${ }^{\circ} 1585$ ).

comme nautonniers à travers toute la région du grand fleuve. Il y a, à Cologne, une stèle inscrite en latin à la mémoire du quartier-maître vigie (proreta) Horus d'Alexandriệ36, et le même musée allemand conserve une autre stèle inscrite à la mémoire d'un pilote (gubernator), Octavius d'Élaia, venu, lui, de Grèce $^{37}$. Nautonniers - tels qu'on le voit sur un relicf du musée de Trèves ${ }^{38}$

(36) Cr. Fritz Fremersdorf, Cologne gallo-romaine el chrétienne, Mémorial d'un voyage de la Soc. nal. des Antiquaires de France en Rhénanie, 1951 (1953), p. 91-136 ; cf. p. 99, fig. 15 : Prorela Alexsandrinus (sic!) ex classe (de la flotte du Rhin, à Cologne).

(37) Ibid., p. 99, fig. 14.

(38) Memorial 1951, l. l., p. 37, fig. 5 ; cf. aussi le célèbre bateau de commerce du Musće de Trèves (bateau sur la Moselle : Neumagen). 
ou commerçants voyageant par mer pour apporter les vins d'Italie, de Gaule, d'Espagne, capilaines de vaisseaux (trierarchi), etc... - tout ainsi qu'à Cologne où le culte d'Isis s'étail implanté ${ }^{39}$ — devaient fréquenter à Strasbourg les parages qui sont ceux de Saint-Étienne aujourd'hui (ancien port de guerre), ct les bassins de commerce reconnus entre la chapelle Saint-Martin et SaintThomas, à l'endroit appelé Rheinecke. Certes, des Alexandrins autres que navi-

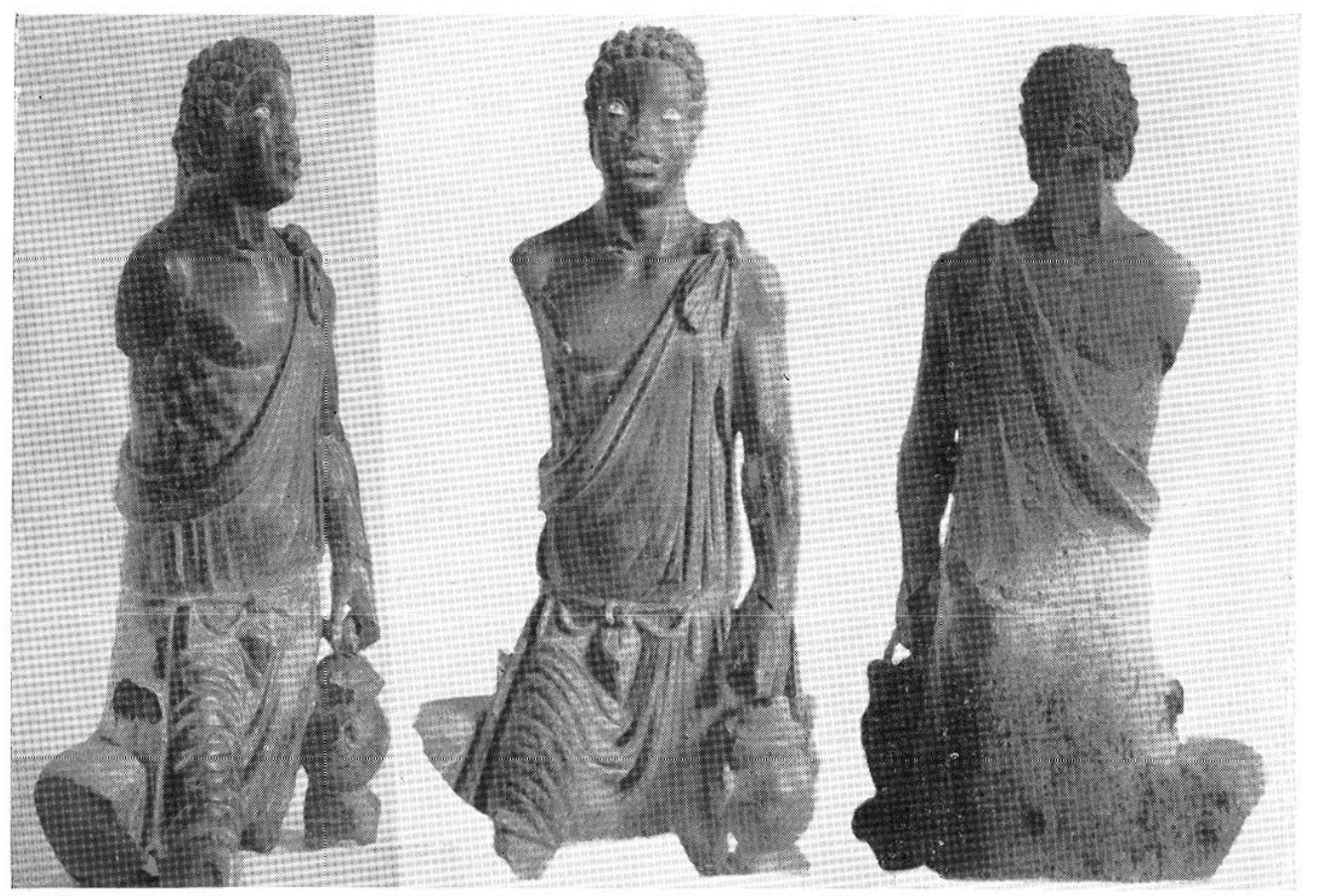

Fig. 5. - Nègre situlophore, d'Aphrodisias en Carie (?).

gateurs avaient bien pu être tentés aussi par l'allrail des richesses de la région rhénane, par les carrières de basalte des entours de Brisach, d'où venaient les meules en lave de la région de Coblence; les bords du Rhin curent aussi on le sait, p. ex., des corporations de verriers" ${ }^{40}$; mais les bateliers habiles du pays du Nil faisaient prime exceptionnellement, rendant les plus grands services pour guider les convois sur un fleuve capricieux ${ }^{41}$; ils durent être, semble-t-il,

(39) Cr. Klaus Parlaska, Die Isis-und Sarapisverehrung um römischen Köln, Kölner Jarsb.f. Vor-u. Frühgesch., I. 1955, p. 18-23; l'A. conclut qu'il faut compter avec des influences venues d'Égypte au lihin, et qui ont agi sur la Cologne romaine.

(40) F. Fremersdorf, l. l., p. 100 (inscription).

(41) P. Charliswortir, Les roules el le lrafic commercial dans l'Empire romain, éd. de Cluny, 1938, p. 45 : découverte, en 1910, d'un bateau naufragé au N.-E. de Strasbourg, avec une cargaison de 800 kilos de meules, venant de Niedermendig à $\Lambda$ rgentorate, vers 275 de notre ère. 
particulièrement appréciés à Argentorate. Ils apportaient d'Orient, avec eux, leurs fétiches et des pacotilles légères.

Je crois devoir ici, en terminant, rappeler l'attention sur le goût de l'exotisme égyptisant qu'on a pu constater souvent en Europe centrale, en IIongrie notamment, et du Rhin au Danube. La navigation fluviale semble aussi l'avoir développé à I ravers cette aire. L'histoire de l'expansion du commerce alexandrin est un grand sujet, qui reste à traiter, et exigera encore beaucoup d'enquêtes de détail. Ce trafic allait du Maroc à l'Extrême-Orient. Il a aidé à faire connaître un peu partout les lypes ethniques que l'Égyple asscmblait; non sculement les nains, mais les nègres ${ }^{42}$. Jc donne ici d'autre part, pour Gallia où elles se trouvent bien à leur place, les pholographies d'une statuette inédite de nègre (fig. 4) du Musée de Pithiviers ${ }^{43}$. Je n'ai pas cru inutile, enfin, de rappeler à l'attention un très remarquable situlophoros (porteur de situle : fig. 5) trop oublié : ce nègre d'Asie Mineurc, un Isiaque peut-être, qui doit provenir des Thermes d'Aphrodisias en Carie, sauf erreur, était une figure d'applique (cf. le revers étayé du col) ; il rappelle ainsi les porteurs de vasque africains des Thermes d'Antonin à Carthage ${ }^{44}$ : et mériterait grandement une étude détaillée. J'hésite encore sur la provenance, et je n'ai pu connaître, malheureusement, la situation actuelle du document ${ }^{45}$. Si le rappel tout provisoire donné ici faisail apparaître de nouveau la pièce, et aidait quelqu'un à en préciser l'origine, la technique, je regretterais moins d'avoir été si peu en état do fournir un meilleur commentaire.

Charles. Picard.

(12) Sur les types de nègres décorant la panse des lagynoi, cf. p. ex. ci-dessus, P. Graindor, p. 87 ; négrillon endormi près d'une amphore, Marg. BIFBre, The sculplure of the Itellenistic Age, 1955, p. 96 .

(43) Cette intéressante statuette, que la photographie n'a pas avantagée, a été trouvée en 1908, dans les sablières de Chilleurs-aux-Bois, et ne paraît pas avoir attiré l'attention jusqu'ici. Je dois les photographies à M. Cl. Perron, directeur du Musée de Pithiviers.

(44) Gilbert Picand, Le monde de Carthage, 1956, pl. 3.

(45) Cf. le silulophoros éthiopien, nu, du Musée du Vatican (Pio Clementino) : S. Rrinach, Rép. slal., I, p. 541 (pl. 883 de Clarac, no 2250). Il est question de refondre actuellement-et la besogne sera de longue haleine - le répertoire, incomplet, et de plus en plus insuffisant, de Grace IIAdiey Beardsley, The negro in Greek and Roman civilization: a sludy of the Ethiopian type, 1929. - Je n'y ai rien trouvé concernant les documents ici reproduits. 